\title{
Treatment of pelvic endometriosis with etonogestrel subdermal implant (Implanon ${ }^{\circledR}$ )
}

\author{
Solomon B Yisa, Angela A Okenwa, Roy P Husemeyer
}

\begin{abstract}
Backgound Endometriosis is one of the commonest benign gynaecological disorders and has a peak incidence between 30 and 45 years of age. Treatment options are medical or surgical, depending on the location and extent of the disease and the woman's childbearing aspirations or need for contraception. Over the past five decades several formulations of progestogen have been used to treat endometriosis.
\end{abstract}

Methods Our study was not planned research but a series of case studies of women with severe symptoms of endometriosis treated in an innovative way with a new long-acting progestogen contraceptive system (etonogestrel subdermal implant) which could reasonably have been expected to have a favourable effect on their disease and its symptoms.

Results Five women with severe pelvic endometriosis were treated. Two of them were nulliparous and wished to become pregnant in due course; the other three had completed their childbearing and were contemplating hysterectomy. One of the five women had relief of pelvic pain but requested removal of the implant after 3 months because of side effects; the other four remained very satisfied with their treatment for the symptoms of pelvic endometriosis. Two of the four very satisfied women have had their implant replaced and the other two are expected to have their implant replaced when necessary.

Conclusion Etonogestrel subdermal implants are an additional treatment option in women with symptoms related to pelvic endometriosis.

J Fam Plann Reprod Health Care 2005; 31(1): 67-70 (Accepted 4 June 2004)

\section{Background}

Endometriosis is one of the commonest benign gynaecological disorders, estimated to be present in $10-25 \%$ of women presenting with gynaecological symptoms in the UK. ${ }^{1}$ The peak incidence is between 30 and 45 years of age. The aetiology of pelvic endometriosis is unknown but the theories of metaplasia (Meyer, 1919) ${ }^{2}$ and retrograde menstruation and implantation (Sampson, $1927)^{3}$ are plausible. The associated risk factors for endometriosis include genital tract outflow anomalies, subfertility, family history and altered immune response.

University Hospitals of Leicester NHS Trust, Leicester Royal Infirmary, Leicester, UK

Solomon B Yisa, MRCOG, Registrar

Angela A Okenwa, MB ChB, Senior House Officer

United Lincolnshire Hospitals NHS Trust, Lincoln County Hospital, Lincoln, UK

Roy P Husemeyer, FRCOG, MFFP, Consultant

Correspondence to: Mr R P Husemeyer, Women and Children's Division, Lincoln County Hospital, Greetwell Road, Lincoln LN2 5QY, UK. E-mail: roy.husemeyer@ulh.nhs.uk
The treatment options are medical or surgical and each modality is influenced by the patient's symptoms, the location and extent of her disease and her childbearing aspirations. Surgical treatment could be conservative, by excision, diathermy cauterisation or laser vapourisation of the endometriosis, or radical by hysterectomy and bilateral oophorectomy. The medical therapies that have been used in the treatment of endometriosis include danazol, gestrinone, gonadotrophin releasing hormone $(\mathrm{GnRH})$ analogues, combined oral contraceptives (COCs) and progestogens. We have previously reported the resolution of endometriotic catamenial haemoptysis in a 36-year-old multiparous woman treated with an etonogestrel subdermal implant (SDI) (Implanon $\left.{ }^{\circledR}\right) .{ }^{4}$ We now report a further five women subsequently treated with etonogestrel SDIs for the management of pelvic endometriosis. A need for contraception was a consideration in the first three women treated but not in the other two. The two women who did not need contraception gave informed consent to treatment for an indication not included in the product licence for the implant.

\section{Case reports}

Case 1

A 30-year-old nulliparous woman was referred with a history of severe dysmenorrhoea for more than 5 years. She had undergone diagnostic laparoscopy at another hospital 4 years previously, which revealed extensive endometriotic deposits in the uterosacral ligaments and pelvic peritoneum. She had been given danazol but stopped this treatment after a few weeks as she considered it ineffective. She then received a subcutaneous injection of goserelin (Zoladex ${ }^{\circledR}$ ) $3.6 \mathrm{mg}$ but declined further $\mathrm{GnRH}$ analogue treatment because of unacceptable side effects.

Abdominal examination was unremarkable, but bimanual pelvic examination revealed tenderness in the posterior vaginal fornix and both adnexa. There was no palpable adnexal mass. Swabs taken for sexually transmitted infections were negative. A pelvic ultrasound scan showed a normal uterus and ovaries.

The options for treating the patient's dysmenorrhoea attributable to endometriosis were discussed. She did not wish to use danazol or a GnRH analogue again and she was unwilling to have another laparoscopy with a view to electrocoagulation of endometriotic foci in the pelvis. She was currently depending on male condom use for contraception and the continuing need for effective contraception led to consideration of a monophasic COC, depot medroxyprogesterone acetate (DMPA) or an etonogestrel SDI. The intention of each of these hormonal methods of contraception was to have been reduction in the frequency or complete suppression of menstruation. She opted for an etonogestrel SDI and this was inserted in February 2000. Irregular light bleeding and brownish discharge followed for several months but she had no other side effects and she did not experience any pelvic pain or dyspareunia. Over the course of the next 3 years she experienced mildly painful periods at intervals of 3-4 months. The dysmenorrhoea was much less severe and the bleeding lighter than before insertion of the implant and she remained content to continue the treatment. The original implant was removed and a new etonogestrel SDI 
inserted in April 2003 (after excluding pregnancy because replacement was 2 months overdue in respect of contraceptive action).

\section{Case 2}

In April 2000, a 31-year-old nulliparous woman underwent laparoscopy for investigation of pelvic pain and dysmenorrhoea of approximately 1 year duration. A preoperative ultrasound scan had shown a left ovarian cyst of $5.7 \mathrm{~cm}$ maximum diameter. Laparoscopy revealed extensive endometriosis in the pouch of Douglas and the uterosacral and broad ligaments. Needle aspiration of the ovarian cyst yielded thick, viscous, dark brown fluid typical of a 'chocolate cyst' (endometrioma). Treatment options including the etonogestrel SDI were discussed. The patient was not sexually active at the time but anticipated re-entering a sexual relationship in the foreseeable future and welcomed the prospect of a treatment that would provide effective contraception. She chose to have an etonogestrel SDI and this was inserted in May 2000. At follow-up 6 weeks later she reported irregular bleeding and occasional headaches. She had not had a period nor had she experienced any pelvic pain since insertion of the implant and she was content to continue the treatment. At further review 3 months later, she complained of persistent irregular vaginal bleeding, increased frequency of headaches, unacceptable weight gain and depression. She had not had a recognised period and she remained free of pelvic pain but the symptoms attributable to side effects of the etonogestrel SDI were intolerable and at her request the implant was removed.

\section{Case 3}

A 37-year-old multiparous woman presented in December 1998 with menorrhagia, severe dysmenorrhoea and midcycle pelvic pain. Pelvic examination revealed a normalsized retroverted uterus with tenderness in the posterior vaginal fornix. Although no adnexal mass was palpable clinically, an ultrasound scan had shown a cystic structure in the left ovary measuring $50 \times 44 \times 42 \mathrm{~mm}$. Initial management was conservative with mefenamic acid for dysmenorrhoea and co-codamol for mid-cycle pain. As the symptoms persisted, a diagnostic laparoscopy was carried out in May 1999. This revealed scattered endometriotic deposits in the pouch of Douglas and uterosacral ligaments and also on the surface of the left ovary, which was firmly adherent to the posterior surface of the left broad ligament. A small endometrioma was visible in the left ovary. The right ovary and Fallopian tube looked normal. After discussion of the treatment options, the patient then received four consecutive injections of goserelin $3.6 \mathrm{mg}$ at 4-week intervals, which resulted in amenorrhoea and complete pain relief. On review in January 2000 she reported two mildly painful periods following completion of the course of goserelin with no mid-cycle pain. She was discharged from follow-up but re-referred in May 2000 with recurrence of symptoms. She now wanted surgical treatment by hysterectomy and bilateral salpingooophorectomy, which had been one of the management options discussed previously. Surgical treatment was agreed in principle but she was also offered an etonogestrel SDI for symptom control and contraception, which she accepted. The SDI was inserted in July 2000. Side effects comprised weight gain, minor irregular vaginal bleeding for 2 months and mild facial acne. She chose to defer further contemplation of surgical treatment and during the course of the following year she had just one period with mild dysmenorrhoea and no pelvic pain. She then failed to keep follow-up appointments but in July 2003 she re- established contact and requested another appointment. When seen she reported complete satisfaction with use of the etonogestrel SDI. She had continued having short episodes of light bleeding, which she assumed were periods, at 3-6-month intervals; her symptoms related to endometriosis remained completely controlled and she had not had any further weight gain or acne. At her request the implant was removed and replaced with a new one.

\section{Case 4}

A 41-year-old woman was referred with a 3-year history of menorrhagia, dysmenorrhoea and pelvic pain. Her symptoms had not improved on treatment with mefenamic acid and tranexamic acid. She had three children and was not using contraception as her husband had undergone a vasectomy. Nothing remarkable was found on clinical examination; however, an ultrasound scan showed an enlarged right ovary containing two echogenic cysts, of maximum diameter 55 and $34 \mathrm{~mm}$, with the ultrasonic appearance of endometriotic cysts. A serum CA 125 assay was moderately raised at $90 \mathrm{kU} / \mathrm{l}$.

Diagnostic laparoscopy, hysteroscopy and uterine curettage were carried out in November 2001. Laparoscopy revealed very extensive endometriosis with widespread peritoneal implants and obliteration of the pouch of Douglas with fixed retroversion of the uterus; the ovaries were adherent to the ipsilateral broad ligaments and the left ovary contained an obvious endometriotic 'chocolate cyst', which was aspirated.

In view of the severity of the patient's symptoms and the observed extent and severity of the endometriosis, treatment options included abdominal hysterectomy and bilateral salpingo-oophorectomy. However, in consideration of the improvement of symptoms related to endometriosis in other women who had recently been given etonogestrel SDIs, this potential treatment was also proposed specifically to relieve her symptoms, as she did not need contraception. She chose to have an etonogestrel SDI on a trial basis and this was inserted in December 2001 on the understanding that surgical treatment remained an alternative option. Irregular bleeding settled within 6 weeks and, at follow-up 3 months later, moderate weight gain was the only side effect attributable to the implant. Because of the dramatic improvement in the symptoms of endometriosis the patient chose to defer surgical treatment. By 18 months after implant insertion she had had just three light and mildly painful periods. She was content to continue etonogestrel SDI treatment indefinitely and it is anticipated that the implant will be replaced after 3 years.

\section{Case 5}

A 41-year-old multiparous woman with known severe pelvic endometriosis was referred with a history of worsening pelvic pain, requesting hysterectomy and bilateral oophorectomy. Over the preceding 18 years she had received numerous different treatments for endometriosis and had undergone two laparotomies for drainage and removal of ovarian endometriomata. On several different occasions the patient had been treated with danazol and the GnRH analogue, buserelin, but she would not contemplate these treatments again. Currently she was taking a monophasic COC for several consecutive cycles at a time to reduce the frequency of very painful withdrawal periods, although she did not need contraception as her husband had had a vasectomy. Treatment with the COC had provided limited improvement in the frequency and severity of pelvic pain but had been associated with troublesome, unscheduled (breakthrough) bleeding. 
Clinical examination revealed mild lower abdominal and pelvic tenderness but nothing else remarkable. An ultrasound scan demonstrated a simple cystic area, $20 \mathrm{~mm}$ in diameter, with no specific characteristics, in the left ovary. The range of treatment options, with which the patient was well acquainted, was discussed and hysterectomy with bilateral oophorectomy was agreed in principle. It was, however, proposed that she should also consider treatment, at least as an interim measure, with an etonogestrel SDI. The patient agreed and had the implant inserted in August 2002. There followed a period of irregular, light, vaginal bleeding for a few weeks but no other side effects and her symptoms of endometriosis were dramatically improved. She decided not to proceed with surgical treatment and remained content to rely on the symptomatic relief she had obtained from the etonogestrel SDI for the foreseeable future.

\section{Discussion}

Our report of a series of patients with pelvic endometriosis treated with etonogestrel SDIs was not planned research. The report relates to the pragmatic treatment of women with severe symptoms of endometriosis in an innovative way with a new contraceptive system that could reasonably have been expected to have a favourable effect on their disease and its symptoms. Important precedents to the treatment of these patients were the several previous reports of effective relief of pain in women with endometriosis treated with progestagens ${ }^{5}$ and our report of the successful treatment of endometriotic catamenial haemoptysis with etonogestrel SDI. ${ }^{4}$

In 1958, Kistner6 first described the use of two progestogens, 17-alpha-hydroxyprogesterone caproate and 17-alpha-ethinyl-17-hydroxy-5(10)estren-3-one (norethynodrel), to treat endometriosis. Subsequently the types of progestogens used have included progesterone derivatives such as medroxyprogesterone acetate, retroprogesterones such as dydrogesterone, and 19nortestosterone derivatives such as norethisterone. They have been given in cyclical regimens or continuously for periods ranging from 3 months to 2 years. ${ }^{5}$ Moghissi and Boyce $^{7}$ claimed $100 \%$ pain relief using $30 \mathrm{mg}$ medroxyprogesterone acetate daily and a similar response was achieved in Johnston's ${ }^{8}$ study of women taking dydrogesterone $5 \mathrm{mg}$ daily. In a prospective, placebocontrolled trial, high-dose medroxyprogesterone acetate $(100 \mathrm{mg} /$ day $)$ was found to compare favourably with danazol. 9 DMPA is the only long-acting progestogen to have been used for treating endometriosis ${ }^{10}$ prior to our report.

The etonogestrel SDI (Implanon) is a single ethylene vinyl acetate copolymer rod containing $68 \mathrm{mg}$ etonogestrel (3-ketodesogestrel). It has extremely high contraceptive efficacy for 3 years, acting primarily by ovulation inhibition, but endometrial suppression, inadequate cervical mucus and an abnormal luteal phase contribute to the effectiveness. ${ }^{11}$ Early studies showed that with serum concentrations of etonogestrel $>90 \mathrm{pg} / \mathrm{ml}$ ovulation was inhibited and concentrations well above $90 \mathrm{pg} / \mathrm{ml}$ are reached within 8 hours of insertion. ${ }^{11}$ Even though ovulation is inhibited, ovarian activity gradually resumes, and by 6 months serum oestradiol and follicle-stimulating hormone concentrations are only slightly lower than preinsertion levels but luteinising hormone surges are prevented..$^{12,13}$

The first three women treated in our series required contraception and could have been offered etonogestrel implants, among a range of other options, whether or not they had symptoms of pelvic endometriosis. Two of the women were nulliparous and intended becoming pregnant in due course; the third had completed her childbearing and had requested treatment for endometriosis by hysterectomy and bilateral salpingo-oophorectomy. The two women who did not require contraception, as their husbands had undergone vasectomy, had been offered and had accepted surgical treatment by abdominal hysterectomy and bilateral salpingo-oophorectomy. For these two women the etonogestrel SDI was offered on a trial basis as a safe, alternative treatment for severe symptoms of endometriosis, although not licensed for that indication.

In only one of the five patients was treatment with an etonogestrel SDI unsuccessful. She had experienced relief of the symptoms of endometriosis but she had persistent vaginal bleeding, frequent headaches, unacceptable weight gain and depression. A review of clinical studies ${ }^{14}$ found that the discontinuation rate for the etonogestrel SDI for contraception was about 5\% per 6 months in the first 18 months. ${ }^{14}$ The main reasons for discontinuation which were probably drug-related were weight increase, acne and headache; other adverse experiences leading to discontinuation included depression, loss of libido and breast symptoms.

Four of the five women in our series have been very satisfied with their treatment with etonogestrel SDIs for the symptoms of pelvic endometriosis. Two of them requested replacement of their implants when the recommended 3year period of efficacy for contraceptive purposes was reached; both of them were relying on the implants for contraception as well as the treatment, of endometriosis. The other two women, who do not require contraception, remain content with their treatment, in each case more than 2 years after insertion of their implants; they are expected to remain content for the foreseeable future, well beyond the recommended 3-year contraceptive efficacy period. Since contraception is not a consideration determining the timing of replacement of their implants, they could be given the option after 3 years of waiting until symptoms return before replacement. These two women, as well as one of the others who did require contraception, have been able to avoid alternative surgical treatment by hysterectomy with bilateral oophorectomy, which all three women had been contemplating. It is reasonably predictable that their symptoms will remain satisfactorily controlled with etonogestrel implants until they have passed the menopause, when treatment will cease to be necessary.

A review of the world literature reveals that our report is the first to describe the use of etonogestrel SDIs for treatment of pelvic endometriosis. Although the cases are anecdotal and not the subject of planned research, the outcomes have been very favourable and a more extensive trial of this therapeutic option is recommended.

Statements on funding and competing interests

Funding. None identified.

Competing interests. None identified.

References

1 Shaw RW. Endometriosis. In: Shaw RW, Soutter WP, Stanton S (eds), Gynaecology (3rd edn). London, UK: Churchill Livingstone, 2003; 510 .

2 Meyer R. Uber den Staude der Frage der Adenomyosites Adenomyoma in Allgemeinen und Insbesondere uber Adenomyosites seroepitheliolis Adenomyometitis Sarcomatosa. Zentralbl Gynakol 1919; 36: 745-759.

3 Sampson JA. Perforating haemorrhagic (chocolate) cysts of the ovary, their importance and especially their relation to pelvic adenomas of the endometrial type. Arch Surg 1927; 3: 245-323.

4 Yisa SB, Okenwa AA, Husemeyer RP. Treatment of endometriotic catamenial haemoptysis with etonogestrel subdermal implant. $\mathrm{Br} J$ Obstet Gynaecol 2004; 111: 385-386.

5 Lindsay PC. Medical and endocrine modulatory treatments. In: Shaw RW (ed.), Endometriosis. Oxford, UK: Blackwell Science, 1994; RW (ed.)

6 Kistner R. The use of newer progestins in the treatment of endometriosis. Am J Obstet Gynecol 1958; 75: 264-278. 
7 Moghissi KS, Boyce CR. Management of endometriosis with oral medroxyprogesterone acetate. Obstet Gynecol 1976; 47: 265-267.

8 Johnston WI. Dydrogesterone and endometriosis. $\mathrm{Br} J$ Obstet Gynaecol 1976; 83: 77-80.

9 Telimaa S, Puolakka J, Ronuberg L, Kauppila A. Placebo-controlled comparison of danazol and high-dose medroxyprogesterone acetate in the treatment of endometriosis. Gynecol Endocrinol 1987; 1: $13-23$.

10 Vercellini P, Di Giorgi O, Oldani S, Cortesi I, Panazza S, Crosignani PG. Depot medroxyprogesterone acetate versus an oral contraceptive combined with very low dose danazol for long-term treatment of pelvic pain associated with endometriosis. Am J
Obstet Gynecol 1996; 175: 396-401.

11 Makarainen L, van Beek A, Tuomivaara L, Asplund B, Bennink HC Ovarian function during the use of a single contraceptive implant: Implanon compared with Norplant. Fertil Steril 1998; 69: 714-721.

12 Croxatto HB, Makarainen L. The pharmacodynamics and efficacy of Implanon. An overview of the data. Contraception 1998; 58: 91S-97S

13 Bennink HJ. The pharmacokinetics and pharmacodynamics of Implanon, a single-rod etonogestrel contraceptive implant. Eur J Contracept Reprod Health Care 2000; 5(Suppl. 2): 12-20.

14 Edwards JE, Moore A. Implanon. A review of clinical studies. $\mathrm{Br} J$ Fam Plann 1999; 24: 3-16.

\section{Journal Club}

Opening a can of worms": GP and practice nurse barriers to talking about sexual health in primary care. Gott $\mathrm{M}$, Galena $\mathrm{E}$, Hinchliff S, Elford H. Fam Pract 2004; 21: 528-536

This study used semi-structured interviews with 22 general practitioners and 35 practice nurses from a variety of general medical practices from a variety of general medical practices across Sheffield (a city in the north of the UK).
The aim was to identify the barriers preventing The aim was to identify the barriers preventing the discussion of sexual health. The participants had particular difficulties discussing sexual health with groups of patients where they did not know how opening up the subject would be received and where they anticipated received and

They identified as particularly difficult: patients of the opposite gender to themselves, patients from ethnic minority groups, middleaged and older patients and non-heterosexual patients. This tells us about the preconceptions of the participants and their lack of training and experience in discussing sexual health.

The other main barrier was the perception that asking about sexual health would 'open a can of worms', that is, that it would reveal information that would take too much time to deal with. Perhaps the description of 'a can of worms' also describes the feeling that 'unsavoury' information would be revealed? The participants felt that discussing sexual health matters in primary care created problems because of the sensitivity and complexity of the material. They also felt constrained by lack of time and expertise.

This small study reinforces other studies showing that the role of primary care in providing sexual health services cannot be expanded without training and education for the people who are to deliver the service. Training and education can remove the anxiety about lack of expertise and help health professionals to learn that almost every patient that they encounter in primary care feels that health professionals should be able to discuss sexual health with them.

Reviewed by Gill Wakley, MD, MFFP Visiting Professor in Primary Care Development, Staffordshire University and Freelance GP, Writer and Lecturer, Abergavenny, $U K$

Hormonal contraceptive use, cervical ectopy, and the acquisition of cervical infections. and the acquisition of cervical infections. Morrison CS, Bright P, Wong EL, Kwok C,
Yacobson I, Gaydos CA, et al. Sex Transm Dis 2004; 31: 561-567

This cohort study was undertaken in Maryland, $\mathrm{MD}$, USA following up three groups of women: two groups initiating hormonal contraception [i.e. depot-medroxyprogesterone acetate (DPMA) or oral contraception (OC)] and a group of controls not using hormonal contraception but attending the same Planned Parenthood clinics for gynaecological care Detailed and carefully thought through exclusion criteria were applied to potential participants in the study, and to time-segments of follow-up. Extensive data were collected on the baseline characteristics of the groups, and pre-existing infections were treated and confirmed cleared before entry to the study. Time-varying risk factors and clinical signs were measured prospectively, and at each follow-up appointment there was high-quality testing of chlamydial infection and careful checking of actual contraceptive use. Sophisticated statistical methods were used to model hazard of cervical infection for each of the two hormonal contraception groups (OC and DPMA), relative to controls (non-hormonal contraception), and to adjust for individual baseline and time-varying covariates.

This analysis showed that young age (15-17 years), two or more sex partners, innercity site of clinic attended, ethnicity (non-white) and DPMA use (relative to nonhormonal contraception) were all statistically significantly associated with increased risk of acquiring cervical infection. The pape concludes that use of DPMA, but not of OC, appears to be associated with increased acquisition of cervical chlamydial an gonoccocal infections. Readers should note and remember the essential qualification 'appears'.

A necessary factor in acquisition of new cervical infection is that one of a woman's sexual contacts is already infected. Behavioura factors can increase the likelihood of thi circumstance (more partners increases the chance of an infected partner) and, where a partner is infected, behaviour such as non-use of condoms will facilitate transmission of the infection. In addition, certain physiological or hormonal factors may mediate acquisition of infection (perhaps youth, hormonal status, ectopy). Conversely, if none of a woman' partners is infected then behavioural and physiological factors are irrelevant. Unbiased comparison of rates of new infection therefore requires similar background pools of infection in the contraceptive-use groups to be compared. in the contraceptive-use groups to be compared.
This was not the case in this study, since the This was not the case in this study, since the
baseline (pre-existing) rate of chlamydia in the DPMA group was nearly three times that in the OC group, and nearly double that in the control group $(8.9 \%, 3.1 \%$ and $4.6 \%$, respectively). No amount of adjusting for facilitatory behavioural or physiological factors can compensate for fundamental differences between the groups in infectious potential. [It is irrelevant whether individual cases of chlamydia detected at baseline were treated and cleared, and were not on the whole re-infected during follow-up. The judgement being made here is about the preexisting level of infection prevalent in the sexual contacts of the group, which is best estimated by infection rates at baseline.]

An observational not a randomised design was chosen because the fact that "most participants attended a clinic to initiate contraception and had a particular contraceptive method in mind" made it neither ethically no practically feasible to randomise. However, the failure to randomise runs a very strong risk of confounding of study findings by factors other than contraceptive use. The crucial potential impact of group-level pool of infection has been considered above, but in addition there were marked differences between the groups in individual behavioural and "physiological' characteristics, at baseline and during followup. The extent and direction of these differences makes it very unlikely that the multivariable adjustment applied will have accounted for al confounding by these individual-leve characteristics. No adjustment was possible to control for pre-existing between-group differences in infection pool.

A further concern about the analysis is that the comparisons made (in the model used) were of DPMA vs controls, and OC vs controls, with no direct test of DPMA vs OC. However, the latter comparison would seem to be the more obvious one to have been made, if wishing to reflect on mechanisms for increased acquisition of infection that are specific to DPMA, which is the thrust of the discussion. The comparisons actually made, of each hormonal group vs controls, are really rather trivial. It would be surprising if initiation of hormonal contraception was not associated with change in behavioural factors facilitating infection. For example, during the follow-up controls did not report a change in prevalence of 'always' using condoms, but both hormonal groups showed a marked reduction in this protective behaviour from at least $30 \%$, down to $13 \%$. Women with infected partners who up to study entry had always used condoms, but stopped once reliable hormonal contraception was initiated, would then become 'at risk' of infection. The extent of this risk in the two hormonal study groups would depend on their pre-existing infection pools, and even more so on the known infection status of current partners, which differed significantly across the two groups.

Three reasons for caution have been noted:

- uncontrolled differences in background infectivity of sexual contacts across the three groups

- marked differences between groups in individual risk factors for infection, to the extent that multivariable adjustment is unlikely to have accounted for all confounding

- failure to compare OC and DPMA directly in the model.

Readers should be aware that for these study results appearances may well be misleading.

Reviewed by Pam Warner, BSc, PhD Senior Lecturer in Medical Statistics, Public Health Sciences, University of Edinburgh Health Sciences, University
Medical School, Edinburgh, UK 